\title{
Predicting the histological invasiveness of pulmonary adenocarcinoma manifesting as persistent pure ground-glass nodules by ultra-high-resolution CT target scanning in the lateral or oblique body position
}

\author{
Hua Ren ${ }^{1,2 \#}$, Fufu Liu ${ }^{2 \#}$, Lei $\mathrm{Xu}^{2}$, Fan $\mathrm{Sun}^{2}$, Jing Cai ${ }^{2}$, Lingwei Yu ${ }^{2}$, Wenbin Guan ${ }^{3}$, Haibo Xiao ${ }^{4}$, \\ Huimin $\mathrm{Li}^{2}$, Hong $\mathrm{Yu}^{1}$ \\ ${ }^{1}$ Department of Radiology, Shanghai Chest Hospital, Shanghai Jiao Tong University, Shanghai, China; ${ }^{2}$ Department of Radiology, Xinhua Hospital, \\ Shanghai Jiao Tong University School of Medicine, Shanghai, China; ${ }^{3}$ Department of Pathology, Xinhua Hospital, Shanghai Jiao Tong University \\ School of Medicine, Shanghai, China; ${ }^{4}$ Department of Cardiothoracic Surgery, Xinhua Hospital, Shanghai Jiao Tong University School of Medicine, \\ Shanghai, China
}

Contributions: (I) Conception and design: H Li; (II) Administrative support: H Li, H Yu; (III) Provision of study materials or patients: H Ren, F Liu, L Xu, F Sun, J Cai, L Yu, W Guan, H Xiao; (IV) Collection and assembly of data: H Ren, F Liu; (V) Data analysis and interpretation: H Ren, F Liu; (VI) Manuscript writing: All authors; (VII) Final approval of manuscript: All authors.

"These authors contributed equally to this work.

Correspondence to: Dr. Hong Yu, MD, PhD. Department of Radiology, Shanghai Chest Hospital Affiliated to Shanghai Jiao Tong University, No. 241 West Huaihai Road, Shanghai 200030, China. Email: yuhongchest@163.com; Dr. Huimin Li, MD, PhD. Department of Radiology, Xinhua Hospital, Shanghai Jiao Tong University School of Medicine, No. 1665 Kongiiang Road, Shanghai 200092, China. Email: lihuimin@xinhuamed.com.cn.

Background: Ultra-high-resolution computed tomography (U-HRCT) has improved image quality for displaying the detailed characteristics of disease states and lung anatomy. The purpose of this study was to retrospectively examine whether U-HRCT target scanning in the lateral or oblique body position (protocol G scan) could predict histological invasiveness of pulmonary adenocarcinoma manifesting as pure groundglass nodules (pGGNs).

Methods: From January 2015 to December 2016, 260 patients with 306 pathologically confirmed pGGNs who underwent preoperative protocol G scans were retrospectively reviewed and analyzed. The U-HRCT findings of preinvasive lesions [atypical adenomatous hyperplasias (AAH) and adenocarcinomas in situ (AIS)] and invasive pulmonary adenocarcinomas [minimally invasive adenocarcinomas (MIA) and invasive adenocarcinomas (IAC)] were manually compared and analyzed using orthogonal multiplanar reformation (MPR) images. The logistic regression model was established to determine variables that could predict the invasiveness of pGGNs. Receiver operating characteristic (ROC) curve analysis was performed to evaluate their diagnostic performance.

Results: There were 213 preinvasive lesions (59 AAHs and 154 AISs) and 93 invasive pulmonary adenocarcinomas (53 MIAs and 40 IACs). Compared with the preinvasive lesions, invasive adenocarcinomas exhibited a larger diameter (13.5 vs. $9.3 \mathrm{~mm}, \mathrm{P}=0.000)$, higher mean attenuation $(-571$ vs. $-613 \mathrm{HU}$, $\mathrm{P}=0.002)$, higher representative attenuation $(-475$ vs. $-547 \mathrm{HU}, \mathrm{P}=0.000)$, lower relative attenuation $(-339$ vs. $-292 \mathrm{HU}, \mathrm{P}=0.000)$ and greater frequencies of heterogeneity $(\mathrm{P}=0.001)$, air bronchogram $(\mathrm{P}=0.000)$, bubble lucency $(\mathrm{P}=0.000)$, and pleural indentation $(\mathrm{P}=0.000)$. Multiple logistic analysis revealed that larger diameter [odds ratio (OR), 1.328; 95\% CI: 1.208-1.461; P=0.000] and higher representative attenuation (OR, 1.005; 95\% CI: 1.003-1.007; $\mathrm{P}=0.000)$ were significant predictive factors of invasive pulmonary adenocarcinomas from preinvasive lesions. The optimal cut-off value of the maximum diameter for invasive pulmonary 
adenocarcinomas was larger than $10 \mathrm{~mm}$ (sensitivity, 66.7\%; specificity, 72.8\%).

Conclusions: The imaging features based on protocol $G$ scanning can effectively help predict the histological invasiveness of pGGNs. The maximum diameter and representative attenuation are important parameters for predicting invasiveness.

Keywords: Lung neoplasms; adenocarcinoma; solitary pulmonary nodule; tomography; X-ray computed

Submitted Dec 20, 2020. Accepted for publication Apr 30, 2021.

doi: 10.21037/qims-20-1378

View this article at: http://dx.doi.org/10.21037/qims-20-1378

\section{Introduction}

Pure ground-glass nodules (pGGNs) are defined as focal increased pulmonary attenuation without solid internal components and with visualization of the bronchial and vascular structures (1), corresponding to different histological types including focal interstitial fibrosis, atypical adenomatous hyperplasias (AAH), adenocarcinoma in situ (AIS), minimally invasive adenocarcinoma (MIA), and occasionally, invasive adenocarcinoma (IAC) (2-4). Due to the inert course and different prognoses, varied management strategies should be reasonably executed by the multidisciplinary team according to different guidelines $(1,5-8)$.

Emerging evidence has shown that sublobar resection with selective mediastinal lymphadenectomy or without lymph node dissection is currently being reconsidered for pGGNs of AIS, MIA, or even invasive adenocarcinoma (2,9-13). Wilshire et al. (14) confirmed that the radiologic classification of patients with lepidic adenocarcinomas in preoperative computed tomography (CT) could guide clinical decision-making in terms of the surgical resection approach of these lesions, as it is very difficult for pathologists to exclude tumor invasion based on intraoperative frozen sections (15). Hence, preoperative radiologic evaluation has an important role to play in patients with pGGNs.

Up to now, visual radiologic assessments based on supine CT imaging have been used to distinguish between preinvasive lesions and invasive pulmonary adenocarcinomas (IPAs) appearing as pGGNs $(3,16)$. Several investigations have suggested nodule size [7.5 mm (17), $10 \mathrm{~mm}$ (3), and $15 \mathrm{~mm} \mathrm{(2)}$ in diameter] and CT density [-293.8 HU in relative CT value (18), $-472 \mathrm{HU}$ in mean CT number (2), and the 100th percentile on histogram (19)] could be used to distinguish between them, while Han et al. (20) and Fu et al. (21) reported the opposite conclusion. Recent studies $(22,23)$ have demonstrated that subsolid nodules classification (pGGNs; heterogeneous GGNs; part-solid nodules) have a significant linear trend with the histological invasiveness degree of the pulmonary adenocarcinoma spectrum. Moreover, radiomics features assessments have also been applied to discriminate histological invasiveness in pGGNs $(24,25)$. However, it is extremely challenging to differentiate these two groups owing to varying criteria and image quality. In this context, there is a pressing need to develop a systematic method for assessing pGGNs. A modified practical technical approach [ultra-high-resolution computed tomography (U-HRCT) target scanning in the lateral or oblique body position (namely protocol G scanning)] to assess pGGNs has been proposed by an earlier investigation (26).

Compared with conventional CT, the main advantage of protocol G scanning is that it provides significantly better image quality for displaying the detailed characteristics of pGGNs through sufficient higher spatial resolution [small $250 \mathrm{~mm}$ field of view (FOV), 1,024×1,024 matrix, $0.24 \mathrm{~mm}$ pixel, and submillimeter slice thickness] and higher contrast resolution (26). The iterative reconstruction technique (iDose4) substantially reduces image noise while maintaining spatial resolution. Lateral or oblique positioning may also be helpful to more fully characterize pGGNs due to hyperinflation of the ipsilateral lung rather than simple deep inspiration (26). Indeed, several previous studies have evaluated pulmonary nodules by using U-HRCT (27-29). However, those studies were limited to evaluating lesions in conventional supine CT scans and transverse planes. In addition, they had not focused on predicting histological invasiveness of pulmonary adenocarcinoma but rather on the assessment of image quality.

Therefore, the purpose of our study was to retrospectively evaluate whether the application of protocol G scanning could predict the histological invasiveness of pulmonary 
adenocarcinoma manifesting as persistent pGGNs.

\section{Methods}

\section{Study population}

This study was conducted following the Declaration of Helsinki (as revised in 2013) and was approved by the Institutional Ethics Committee of Xinhua Hospital affiliated to Shanghai Jiao Tong University School of Medicine. Individual consent for this retrospective analysis was waived. Study subjects who had undergone protocol G scans from January 2015 to December 2016 at our institution were reviewed via the radiology information systems. The inclusion criteria were as follows: (I) patients with persistent pGGNs $\leq 30 \mathrm{~mm}$ in the longest diameter on CT axial images (lesions with a diameter $\leq 5 \mathrm{~mm}$ on CT images usually do not warrant surgical treatment in our hospital); and (II) availability of submillimeter thin slice preoperative CT images. The exclusion criteria were as follows: (I) patients with solid or part-solid nodules; (II) patients with lesions greater than $3 \mathrm{~cm}$; (III) cases with no thin-section preoperative CT images available; (IV) lesions in emphysematous lung regions; (V) motion artifacts; (VI) pathologically described lesion not identifiable on CT; and (VII) inflammatory lesions.

According to the 2015 World Health Organization classification of lung tumors (30), preinvasive lesions for lung adenocarcinoma were defined as lesions showing no stromal, vascular, or pleural invasion, comprising AAH and AIS. IPAs included MIA and IAC (lepidic predominant, acinar predominant, or predominant papillary adenocarcinoma) and were defined as adenocarcinomas that included an invasive component.

\section{CT examination}

Protocol G scans were performed on all patients using a Philips Brilliance 256 iCT scanner (Philips Healthcare, Haifa, Israel) by an experienced technician (J. C., with 24 years of experience in CT scanning). The scans were performed at full inspiration and without intravenous contrast material injection, which makes locating lesions in the higher area of the scanned lung as much as possible, and ensures that the lesion and adjacent lung parenchyma are fully inflated. The scan parameters were as follows: $120 \mathrm{kV}$; $250 \mathrm{mAs}$; collimation, $0.625 \mathrm{~mm} \times 64 \mathrm{~mm}$; ultra-high-resolution mode: FOV, $250 \mathrm{~mm}$; and matrix,
$1,024 \times 1,024$. Reconstruction was carried out with $2.5 \mathrm{~mm}$ thickness using a filter back projection and high-resolution algorithm without interval. Retrospective reconstruction was performed by reducing the FOV with iterative reconstruction at iDose level 4: section thickness, $0.67 \mathrm{~mm}$; interval, $0.67 \mathrm{~mm}$; smooth (A) filter lung kernel. After conventional CT examination of the entire thorax in our hospital or other institutions, protocol G scans were performed independently from the previous CT scans with a new limited-range scan of only the lesions to reduce additional radiation exposure, and different patient positioning was employed to more accurately evaluate lesions for a follow-up CT examination or further surgical treatment. For patients with multiple pulmonary nodules, protocol G scans were performed based on the dominant or most suspicious nodule. The CT radiation dose, volume CT dose index (mean, 21.84 $\pm 1.24 \mathrm{mGy}$ ), and dose-length product (mean, $296.31 \pm 63.29 \mathrm{mGy} \cdot \mathrm{cm}$ ) were recorded. Effective dose (mean, $4.14 \pm 0.89 \mathrm{mSv}$ ) was calculated by multiplying the dose-length product with the $\mathrm{k}$ conversion coefficient $[0.014 \mathrm{mSv} /(\mathrm{mGy} \cdot \mathrm{cm})]$ for chest CT.

\section{Evaluation of CT features}

The post-reconstructed images were transferred to the Philips Extended Workspace post-processing workstation (IntelliSpace Portal, V6.0.6.20039, Philips Healthcare, Haifa, Israel) and were used to manually accomplish multiplanar reformation (MPR), including $0.67 \mathrm{~mm}$ thickness, $3 \mathrm{~mm}$ thickness, and three-dimensional volume rendering based on bronchial vascular bundles and pleura. Meanwhile, morphological characteristics and relationships with the adjacent bronchi and blood vessels of the lesions were displayed.

The final preoperative CT images were collected and assessed. The CT features of pGGNs were retrospectively analyzed by two observers (H. R. and H. M. L., with 4 and 30 years of experience in chest imaging, respectively) who were unaware of the pathological results. In cases of discrepant interpretations, a consensus was achieved by discussion. A persistent pGGN was defined as purely of ground-glass opacity on lung window images (width, $1,500 \mathrm{HU}$; level, $-500 \mathrm{HU}$ ) that had no soft-tissue attenuation within the lesion on the mediastinal window images (width, $400 \mathrm{HU}$; level, $40 \mathrm{HU}$ ), which remained stable or grew in size during the follow-up examination period of 3 months or longer. The Maximum diameter and attenuation were manually measured and analyzed 

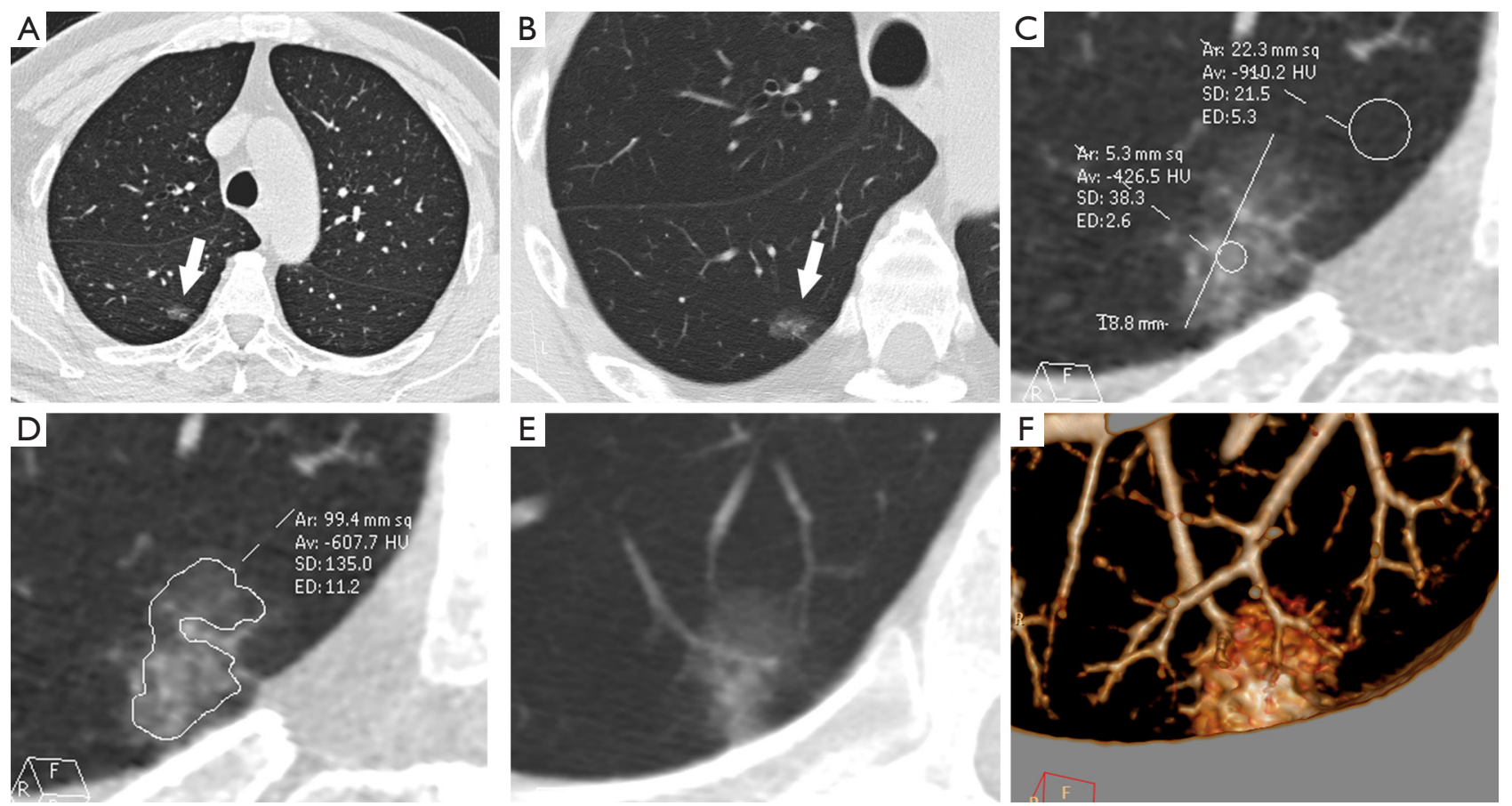

Figure 1 Conventional CT scan and U-HRCT target scan in the lateral or oblique body position (protocol G scan) of pulmonary adenocarcinoma appearing as pGGNs. Conventional CT in the supine position (A) and protocol G scan in the left lateral position (B) show an $11 \mathrm{~mm}$ heterogenous pGGN (arrow) with well-defined border in the right lower lobe of a 60-year-old man. (C) The maximum diameter of this nodule was $19 \mathrm{~mm}$ after orthogonal MPR, representative attenuation of this nodule was $-426 \mathrm{HU}$, and CT attenuation of normal lung parenchyma was -910 HU. (D) The mean attenuation of this nodule was -608 HU, relative attenuation of this nodule was -302 HU. (E) Thickness MPR (section thickness, $3 \mathrm{~mm}$ ). (F) Three-dimensional volume rendering. This nodule was confirmed as invasive adenocarcinoma at sublobar resection. CT, computed tomography; U-HRCT, ultra-high-resolution computed tomography; pGGN, pure ground-glass nodule; MPR, multiplanar reformation.

on the maximal section of the pulmonary nodules in orthogonal MPR images. The CT findings of each nodule were analyzed, and included the following parameters: (I) maximum diameter; (II) attenuation: mean attenuation [an oval or irregular region of interest (ROI) $\geq 2 \mathrm{~mm}$ in diameter, covering $80 \%$ of the largest area in a lesion away from air containing space and large vessels], representative attenuation (a ROI $\geq 2 \mathrm{~mm}$ in diameter, covering the higher attenuation area in a lesion was selected), CT attenuation of normal lung parenchyma (a ROI in normal lung parenchyma closing the lesion, approximately $5 \mathrm{~mm}$ in diameter, was selected), and relative attenuation (CT attenuation of normal lung parenchyma minus mean attenuation); (III) homogeneity (homogeneous opacity pGGNs viewed using the lung window) or heterogeneity (heterogeneous pGGNs with irregular areas with low and high attenuation in only the lung window but not in the mediastinal window) $(31,32)$; (IV) shape (round or oval and irregular), (V) tumor- lung interface (well-demarcated or ill-demarcated); (VI) air bronchogram; (VII) bubble lucency; and (VIII) pleural retraction. Air bronchograms were defined as branching or tubular air-filled bronchi within the nodule. Bubble lucency was described as air attenuation vesicle-like lucency within the nodule. The pleural retraction was depicted as a retraction of the adjacent pleura toward the nodule. Representative images are shown in Figure 1.

In addition, to evaluate the interobserver agreement of CT features of pGGNs, one chest radiologist (F. F. L., with 4 years of experience in chest imaging) independently analyzed the CT features of pGGNs and was blinded to the pathological results of the pGGNs.

\section{Statistical analysis}

All statistical analyses were performed using SPSS software (version 24; IBM, Armonk, NY). We used the Wilcoxon 


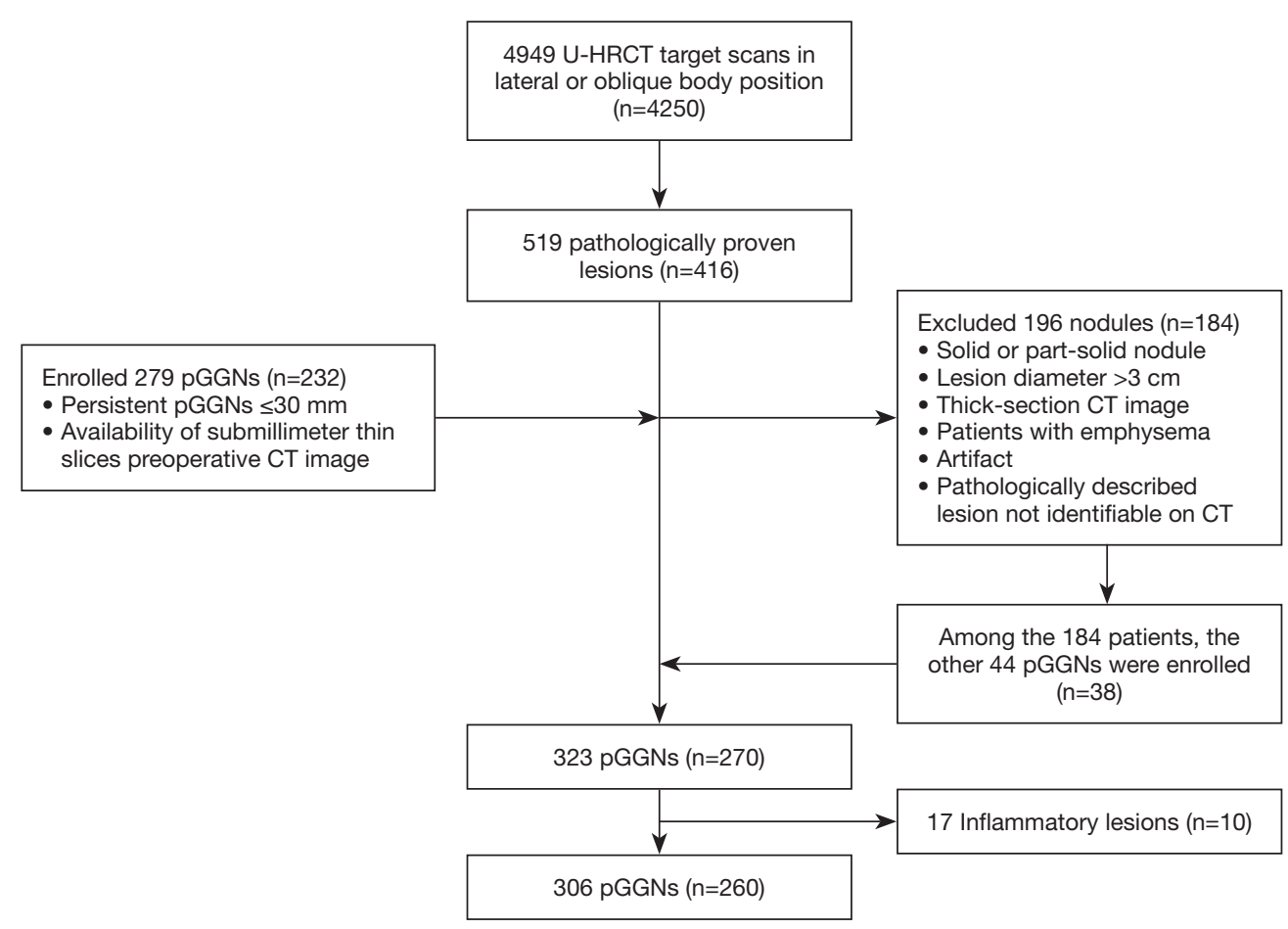

Figure 2 Flow diagram of patient inclusion and exclusion. CT, computed tomography; U-HRCT, ultra-high-resolution computed tomography; pGGNs, pure ground-glass nodules.

rank-sum test to compare differences in the maximum diameter and used the independent sample $t$-test to compare differences in attenuation between preinvasive lesions and IPAs. Statistical comparisons of morphological features of these two groups were analyzed using Pearson $\chi^{2}$ test or Fisher's exact test.

Receiver operating characteristic (ROC) curve analysis was also used to compare the abilities of mean attenuation, representative attenuation, and relative attenuation to differentiate IPAs from preinvasive lesions and was performed to determine the optimal cut-off values and differentiate the performance of significant continuous variables. The Youden index was used to determine the optimal cutoff point.

Variables that exhibited statistically significant differences between preinvasive lesions and IPAs were used as the input variables for multiple logistic regression. A backward stepwise feature selection strategy was used in multiple logistic regression to assess the relationship between CT characteristics of pGGNs and lesion invasiveness. ROC curves were used to evaluate the performance of the logistic regression model for predicting invasiveness.

Interobserver agreement regarding CT features of pGGNs for quantitative variables was assessed using linear regression analysis. Weighted kappa statistics for qualitative features and classified as poor $(\kappa=0.00-0.20)$, fair $(\kappa=0.21$ $0.40)$, moderate $(\kappa=0.41-0.60)$, good $(\kappa=0.61-0.80)$, or excellent $(\kappa=0.81-1.00) . \mathrm{P}<0.05$ was considered to indicate a statistically significant difference.

\section{Results}

\section{Patient and nodule characteristics}

From January 2015 to December 2016, a total of 4,949 protocol $\mathrm{G}$ scans in 4,250 patients were obtained by our radiology information systems. Among these patients, 416 consecutive patients with 519 pathologically proved lesions who underwent curative resection of lung adenocarcinoma in our hospital were reviewed via the Department of Thoracic Surgery medical records and pathological systems. The detailed selection algorithm is summarized in Figure 2. Our study population consisted of 260 patients (median age, 53 years; range, 15-82 years) with 306 pGGNs diagnosed by pathological analysis. There were 70 men (median age, 51 years; range, 24-77 years) and 190 women (median age, 54 years; range, $15-82$ years). Among the 260 patients, 223 
Table 1 Baseline clinicopathologic characteristics of study objects

\begin{tabular}{|c|c|}
\hline Characteristic & Result (\%) \\
\hline Age, years, median (range) & 53 [15-82] \\
\hline \multicolumn{2}{|l|}{ Sex } \\
\hline Male & $70(26.9)$ \\
\hline Female & $190(73.1)$ \\
\hline \multicolumn{2}{|l|}{ Symptoms } \\
\hline Cough, sputum & $32(12.3)$ \\
\hline Chest pain & $14(5.3)$ \\
\hline Hemoptysis & $2(0.8)$ \\
\hline Fever & $1(0.4)$ \\
\hline Hoarseness & $1(0.4)$ \\
\hline No symptoms & $210(80.8)$ \\
\hline \multicolumn{2}{|l|}{ Smoking status } \\
\hline Non-smoker & $239(91.9)$ \\
\hline Former smoker & $8(3.1)$ \\
\hline Current smoker & $13(5.0)$ \\
\hline \multicolumn{2}{|l|}{ Surgery } \\
\hline Lobectomy & $71(27.3)$ \\
\hline Sublobar resection & $163(62.7)$ \\
\hline Lobectomy with sublobar resection & $16(6.1)$ \\
\hline \multicolumn{2}{|l|}{ Bilateral pulmonary resections } \\
\hline Lobar-lobar & $1(0.4)$ \\
\hline Lobar-sublobar & $2(0.8)$ \\
\hline Sublobar-sublobar & $7(2.7)$ \\
\hline Lymph node metastasis & $1(0.4)$ \\
\hline Pleural invasion & $7(2.7)$ \\
\hline \multicolumn{2}{|l|}{ Location } \\
\hline Right upper lobe & $109(35.6)$ \\
\hline Right middle lobe & $27(8.8)$ \\
\hline Right lower lobe & $41(13.4)$ \\
\hline Left upper lobe & $83(27.1)$ \\
\hline Left lower lobe & $46(15.0)$ \\
\hline \multicolumn{2}{|l|}{ Pathologic subtype } \\
\hline AAH & $59(19.3)$ \\
\hline AIS & $154(50.3)$ \\
\hline MIA & 53 (17.3) \\
\hline IAC & $40(13.1)$ \\
\hline
\end{tabular}

$\mathrm{AAH}$, atypical adenomatous hyperplasia; AIS, adenocarcinoma in situ; MIA, minimally invasive adenocarcinoma; IAC, invasive adenocarcinoma. had single pathologically proved pGGNs (38 AAHs, 113 AISs, 40 MIAs, and 32 IACs). The remaining 37 patients had multiple pathologically proved pGGNs (two patients had two AAHs, seven patients had two AISs, two patients had three AISs, two patients had two MIAs, seven patients had one AAH and one AIS, two patients had one AAH and one MIA, five patients had one AIS and one IAC, four patients had one AIS and one MIA, three patients had one AAH, one AIS, and one MIA, one patient had two AAHs and one AIS, one patient had three AAHs and one IAC, and one patient had one AIS and two IACs).

Among the 260 patients, 71 patients underwent lobectomy, 163 underwent sublobar resection (wedge resection or segmentectomy), and 16 underwent lobectomy with sublobar resection. Bilateral lobectomy was performed in one case, lobectomy and contralateral sublobar resection were carried out in two cases, and bilateral sublobar resections were conducted in the remaining seven cases. One had a $3 \mathrm{~cm}$ solid mass in the right upper lobe in all patients but had a pGGN in the left upper lobe had lymphatic metastasis, and another seven had a pleural invasion. The mean interval between the latest $\mathrm{CT}$ and surgery was 14 days (median, 9 days; range, 1-172 days).

In our study, among the 306 pathologically proved pGGNs, there were 213 preinvasive lesions (59 AAHs and 154 AISs) and 93 invasive lesions (53 MIAs and 40 IACs). Detailed patient and nodule characteristics are described in Table 1.

\section{Comparison of CT features between preinvasive lesions and IPAs}

Detailed results of the CT characteristics are summarized in Table 2. Compared with preinvasive lesions, IPAs exhibited a larger diameter (13.5 vs. $9.3 \mathrm{~mm}, \mathrm{P}=0.000)$, higher mean attenuation ( -571 vs. $-613 \mathrm{HU}, \mathrm{P}=0.002)$, higher representative attenuation $(-475 v s$. $-547 \mathrm{HU}, \mathrm{P}=0.000)$, lower relative attenuation ( $-339 v s .-292 \mathrm{HU}, \mathrm{P}=0.000)$ and greater frequencies of heterogeneity $(\mathrm{P}=0.001)$, air bronchogram $(\mathrm{P}=0.000)$, bubble lucency $(\mathrm{P}=0.000)$, and pleural indentation $(\mathrm{P}=0.000$; Figures 3,4$)$. The $\mathrm{CT}$ attenuation of normal lung parenchyma and tumor-lung interface was not significantly different between the two groups $(\mathrm{P}>0.05)$.

\section{Logistic regression analysis and $R O C$ analysis}

For predicting invasiveness, a higher area under the curve (AUC) and specificity could be achieved by using 
Table 2 CT features of preinvasive lesions and IPAs in pGGNs

\begin{tabular}{|c|c|c|c|}
\hline Characteristic & Preinvasive lesions (\%) & IPAs (\%) & $P$ value \\
\hline Maximum diameter $(\mathrm{mm})^{*}$ & $9.3 \pm 2.8$ & $13.5 \pm 5.7$ & 0.000 \\
\hline \multicolumn{4}{|l|}{ Attenuation $(\mathrm{HU})^{*}$} \\
\hline Mean attenuation & $-613 \pm 110$ & $-571 \pm 99$ & 0.002 \\
\hline Relative attenuation & $-292 \pm 106$ & $-339 \pm 95$ & 0.000 \\
\hline Normal lung parenchyma & $-905 \pm 22$ & $-910 \pm 22$ & 0.055 \\
\hline Shape & & & 0.252 \\
\hline Oval & 202 (94.9) & $85(91.4)$ & \\
\hline Homogeneity & $36(16.9)$ & $3(3.2)$ & \\
\hline Heterogeneity & $177(83.1)$ & $90(96.8)$ & \\
\hline Tumor-lung interface & & & 0.597 \\
\hline Well-demarcated & $193(90.6)$ & $86(92.5)$ & \\
\hline III-demarcated & $20(9.4)$ & $7(7.5)$ & \\
\hline Air bronchogram & $20(9.4)$ & $31(33.3)$ & 0.000 \\
\hline Bubble lucency & $7(3.3)$ & $17(18.3)$ & 0.000 \\
\hline Pleural retraction & $25(7.5)$ & $16(26.9)$ & 0.000 \\
\hline
\end{tabular}

${ }^{*}$ Data are presented as means \pm standard deviation. CT, computed tomography; pGGNs, pure ground-glass nodules; IPAs, Invasive pulmonary adenocarcinomas.

representative attenuation (AUC $=0.652$, specificity: $68.1 \%$ ) than mean attenuation (AUC $=0.619$, specificity: $63.4 \%$ ) or relative attenuation (AUC $=0.643$, specificity: $62.9 \%$; Table S1).

The maximum diameter, representative attenuation, homogeneity, air bronchogram, bubble lucency, and pleural indentation were used as input variables for multiple logistic regression analysis. Logistic regression analysis revealed that larger diameter [odds ratio (OR), 1.328, 95\% CI: 1.208 1.461, $\mathrm{P}=0.000]$ and higher representative attenuation (OR, 1.005 , 95\% CI: $1.003-1.007, \mathrm{P}=0.000$ ) were significantly associated with the IPAs (Table 3).

ROC analysis revealed that the AUC for the maximum diameter was 0.764 (95\% CI: 0.706-0.821). The optimal cut-off value for the maximum diameter differentiating IPAs from previous lesions was larger than $10 \mathrm{~mm}$ (sensitivity: $66.7 \%$, specificity: $72.8 \%$ ).

\section{Performance of logistic regression model}

ROC analysis was conducted to evaluate the performance of the logistic regression model by using the maximum diameter and representative attenuation for predicting histological invasiveness, and the AUC was 0.791 (95\% CI: $0.736-0.847)$. The sensitivity and specificity of the logistic regression model were $55.9 \%$ and $86.9 \%$, respectively. The performance of the logistic regression model was markedly higher than that using only the maximum diameter or representative attenuation alone (Figure 5).

\section{Interobserver agreement for CT findings of $p G G N s$}

The interobserver agreement results regarding the CT features of the pGGNs are summarized in Table S2. Linear regression analysis for the maximum diameter was $y=0.359$ 

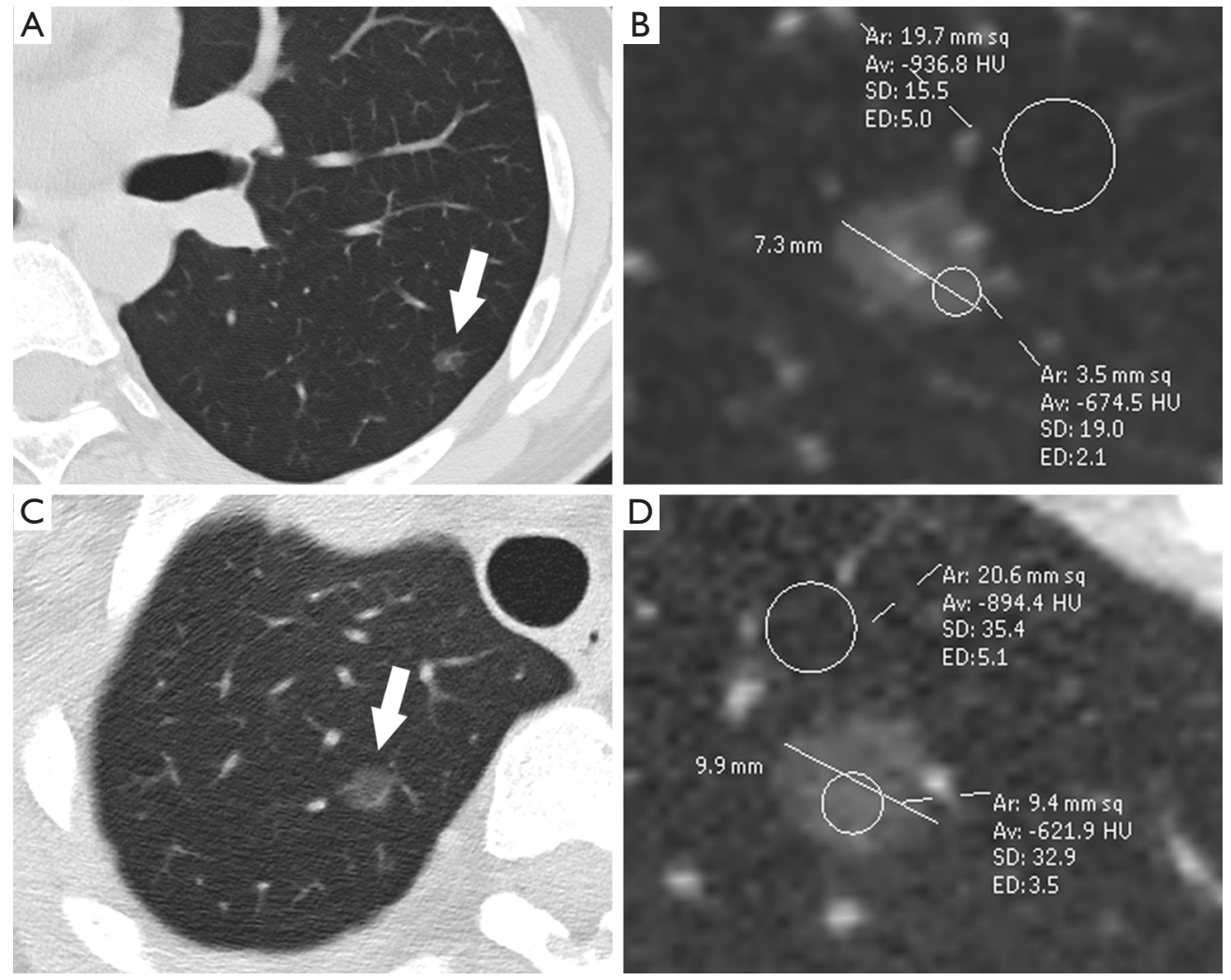

Figure 3 U-HRCT target scans of preinvasive lesions appearing as pGGNs. (A,B) A homogeneous pGGN (arrow) in the left upper lobe of a 37-year-old woman. The maximum diameter and representative attenuation of this nodule were $7 \mathrm{~mm}$ and $-675 \mathrm{HU}$, respectively. This nodule was confirmed as AAH upon sublobar resection. (C,D) A homogeneous pGGN (arrow) in the right upper lobe of a 38-yearold woman. The maximum diameter and representative attenuation of this nodule were $10 \mathrm{~mm}$ and -622 HU, respectively. This nodule was confirmed as AIS upon sublobar resection. U-HRCT, ultra-high-resolution computed tomography; pGGNs, pure ground-glass nodules; AAH, atypical adenomatous hyperplasia; AIS, adenocarcinoma in situ.

$+0.997 x\left(\mathrm{R}^{2}=0.966, \mathrm{P}=000\right)$. Linear regression analysis for mean, representative attenuation, or CT attenuation of normal lung parenchyma were $y=-32.712+0.940 x$ $\left(\mathrm{R}^{2}=0.790, \mathrm{P}=000\right), y=-16.389+0.911 x\left(\mathrm{R}^{2}=0.779, \mathrm{P}=000\right)$, and $y=-150.040+0.833 x\left(\mathrm{R}^{2}=0.660, \mathrm{P}=000\right)$, respectively. Interobserver agreement for other features showed moderate to good agreement. The $\kappa$ values ranged from 0.418 to 0.711 .

\section{Discussion}

The evaluated criteria of pGGNs, remain controversial and have not been completely agreed upon because of substantial overlaps between the imaging features of histopathologic types and different assessment methods. To our knowledge, there are no studies that have attempted to investigate imaging features and predict histological invasiveness via protocol G scanning. Our results showed that larger diameter and larger representative attenuation were independently associated with IPAs.

In clinical practice, the nodule dimension is the key CT parameter for management recommendations and oncologic staging (4-8). In this study, we found that the maximum diameter for discriminating IPAs from preinvasive lesions appearing as pGGNs was $10 \mathrm{~mm}$, according to previous literature. Lee et al. (3) showed that a lesion size of less than $10 \mathrm{~mm}$ to differentiate preinvasive lesions from IPAs demonstrated a sensitivity of $53.33 \%$ and specificity of $100 \%$. Chu et al. (33) also reported that the optimal cutoff value to differentiate AAH, AIS, and MIA from IA was $10.5 \mathrm{~mm}$. However, the measurement methods varied between previous research and our study; the former measured the maximum dimension on the conventional transverse plane, while the latter measured 

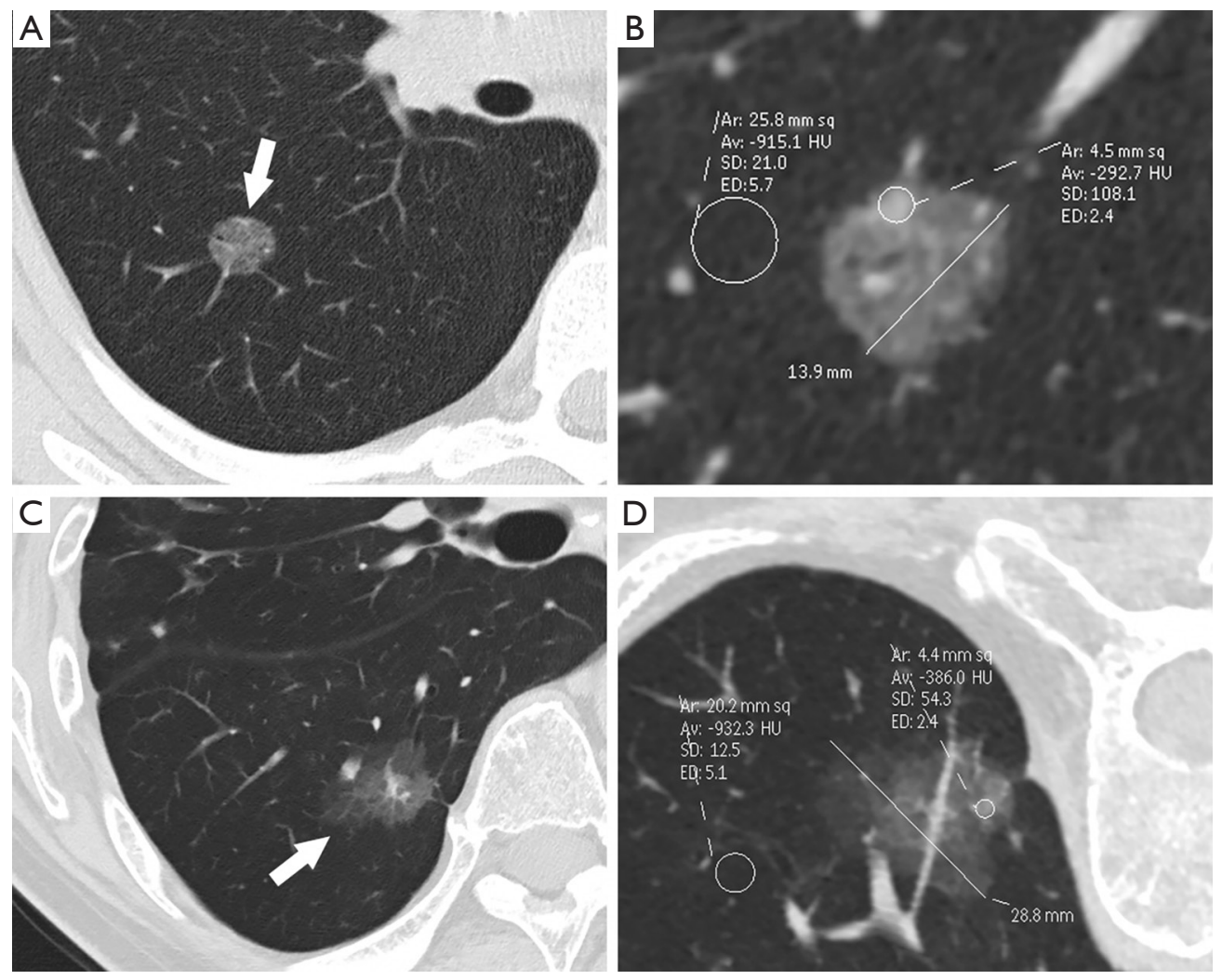

Figure 4 U-HRCT target scans of IPAs appearing as pGGNs. (A,B) A heterogeneous pGGN (arrow) in the right upper lobe of a 32-yearold woman. The maximum diameter and representative attenuation of this nodule were $14 \mathrm{~mm}$ and $-292 \mathrm{HU}$, respectively. This nodule was confirmed as MIA upon sublobar resection. (C,D) A heterogeneous pGGN (arrow) with pleural retraction in the right lower lobe of a 77-year-old woman. The maximum diameter and representative attenuation of this nodule were $29 \mathrm{~mm}$ and $-386 \mathrm{HU}$, respectively. This nodule was confirmed as IAC upon sublobar resection. U-HRCT, ultra-high-resolution computed tomography; pGGNs, pure ground-glass nodules; IPAs, Invasive pulmonary adenocarcinomas; MIA, minimally invasive adenocarcinoma; IAC, invasive adenocarcinoma.

Table 3 Logistic regression analysis for predicting the histological invasiveness of pGGNs

\begin{tabular}{lccc}
\hline Variable & OR & $95 \% \mathrm{Cl}$ & $\mathrm{P}$ value \\
\hline Maximum Diameter & 1.328 & $1.208-1.461$ & 0.000 \\
Representative attenuation & 1.005 & $1.003-1.007$ & 0.000 \\
\hline
\end{tabular}

pGGNs, pure ground-glass nodules; OR, odds ratio; $\mathrm{Cl}$, confidence interval.

the maximum dimension on the orthogonal MPR images manually. Although off-axis oblique reformations are not recommended by the Fleischner society (6), orthogonal MPR can reflect the true invasive extent and obtain a more accurate assessment of nodule size. However, determining the optimal measurement method that can be better used to predict invasiveness requires further study.

Attenuation is another valuable factor in assessing pGGNs. As we know, the density of the lung is affected rapidly and markedly by the degree of aspiration. Gravity causes regional differences in density by decreasing alveolar expansion and increasing the vascular component in dependent areas (34,35). Pennati et al. (36) pointed out that a strong dependence on gravity was found in density variations, with greater values in the dorsal regions of the lung. A change from the supine to the prone position can eliminate the effect of lung volume and gravity on the attenuation and size of pGGNs and helps to narrow the 


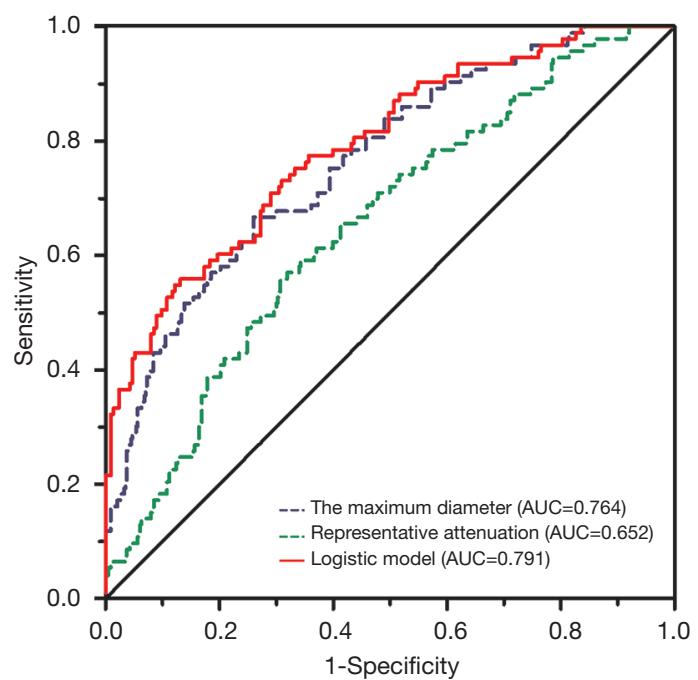

Figure 5 ROC curve analysis of the maximum diameter, representative attenuation, and logistic regression model in predicting the histological invasiveness of pulmonary adenocarcinoma appearing as pGGNs. The AUC of the logistic model including the maximum diameter and representative attenuation was significantly higher (AUC $=0.791$ ) compared to using only the maximum diameter ( $\mathrm{AUC}=0.764$ ) or representative attenuation ( $\mathrm{AUC}=0.652$ ) alone. ROC, Receiver operating characteristic. pGGNs, pure ground-glass nodules; AUC, area under the curve.

differential diagnosis of ground-glass opacity (37). Thus, to remove the gravitational effect of the routine CT, the lateral or oblique body position was adopted in all patients, locating which makes locating lesions in the higher area of the scanned lung as much as possible, and ensures that the lesion and adjacent lung parenchyma are fully inflated.

Some studies have also used the CT value to evaluate the growth of non-solid nodules and demonstrate that an increase in mean attenuation of the non-solid nodule reflects a stepwise progression of adenocarcinoma (38-40). Zhao et al. (41) introduced the concept of relative density, which is calculated by comparing the CT attenuation of the lesion to the normal lung tissue. However, unlike most previous studies, in our study, relative attenuation was calculated by CT attenuation of normal lung parenchyma minus mean attenuation. Relative attenuation can reduce the gravitational effect and be used to assess the invasiveness of the lesion according to varied inhalation degrees between different individuals. Moreover, representative attenuation was proposed in the present study. A higher AUC and specificity could be achieved by using representative attenuation than mean or relative attenuation. Representative attenuation corresponds to the higher attenuation area in a lesion, which may be correlated with the invasive foci of a lesion (42). Regarding the measurement standardization and application of attenuation, more evidence is required.

Concerning the diagnostic performance of protocol $\mathrm{G}$ scanning in predicting the histological invasiveness of pGGNs, the logistic regression model exhibited a significantly higher diagnostic performance compared to using only the maximum diameter or representative attenuation alone. To some extent, the predictive model's performance based on protocol $\mathrm{G}$ scanning is fair and similar to routine CT scanning. These differences could be attributed to several causes, including study design and varying visual assessment. Firstly, the size, density, and morphological CT features of pGGNs were affected by different body positions due to respiratory and gravitational effects. Secondly, size and attenuation play important roles in routine practice. Pre-surgical evaluation by a combination of size and attenuation could improve the identification of invasiveness of pGGNs. However, some previous studies have shown that only one of either size or density is a significant differentiator in the predictive model $(3,33)$. Moreover, we put forward the concept of representative density. Our study provides promising preliminary evidence into the potential clinical applications of attenuation measurement as a tool to assess pGGNs more accurately. Thirdly, the grouping method or different inclusion criteria for pGGNs varied. In this study, a pGGN was identified on CT images with mediastinum window settings. In contrast, some studies defined pGGNs with lung window settings (33) or grouped pGGNs as IAC and AAH-MIA (43). Fourthly, a further large-scale study for evaluating the difference between conventional CT in the supine position and protocol G scanning should be conducted. We believe that protocol $\mathrm{G}$ scanning may be helpful in comprehensively displaying the characteristics of pGGNs.

Several studies $(24,25)$ have been conducted and have developed models based on radiomics parameters to predict the histological invasiveness of pGGNs, which demonstrated satisfactory performance. However, we aimed to provide some initial insights based on protocol $\mathrm{G}$ scanning that can predict the histological invasiveness of pGGNs in clinical practice. The radiomics method is a complex and time-consuming process. In addition, the extracted image features were subjectively handcrafted 
radiomics features. Hence, it is inconvenient for physicians to facilitate preoperative examination. To accurately assess pGGNs, we expect that the G protocol can serve as an easy-to-use and effective tool and that the combination of the $\mathrm{G}$ protocol and radiomics analysis could improve the predictive value in future studies.

Some limitations in the present study should be mentioned. Firstly, due to this study's retrospective singlecenter nature, some selection bias may be unavoidable since only surgically resected and pathological confirmed pGGNs were enrolled. Thus, it is necessary to verify the present results with multi-institutional data and larger sample size. Secondly, some morphological characteristics showed moderate to good agreement, which can most likely be attributed to subjective and manual estimates in the process of orthogonal MPR images (off-axis oblique reformations) instead of a traditional transverse plane or the lack of adequate experience. This aspect needs to be further improved. Thirdly, all scans and data used in our study were obtained on available workstations provided by only one company, and therefore, it is unclear whether workstations provided by other CT vendors will exhibit similar results. Thus, further validation studies are required.

\section{Conclusions}

The imaging features based on protocol G scanning can effectively help to predict the histological invasiveness of persistent pGGNs. The maximum diameter and representative attenuation were important parameters for predicting invasiveness.

\section{Acknowledgments}

The abstract has been accepted for oral presentation at the European Congress Radiology 2020.

Funding: This work was supported by the National Natural Science Foundation of China [grant number 81671679 , 81871353 and 82071873], the 2018 Xinhua-uOttawa Joint Clinical Research Project [grant number 18JXO07], and the AI Cooperation Project of Hospital and Xuhui District [grant number 2020-010].

\section{Footnote}

Conflicts of Interest: All authors have completed the ICMJE uniform disclosure form (available at http://dx.doi. org/10.21037/qims-20-1378). Dr. HL reports that he received a grant from the 2018 Xinhua-uOttawa Joint Clinical Research Project [grant number 18JXO07]; Dr. HY reports that she received grants from the National Natural Science Foundation of China [grant number 81671679, 81871353, and 82071873] and from the AI Cooperation Project of Hospital and Xuhui District [grant number 2020010]. The remaining authors have no conflicts of interest to declare.

Ethical Statement: The authors are accountable for all aspects of the work in ensuring that questions related to the accuracy or integrity of any part of the work are appropriately investigated and resolved. This study was conducted in accordance with the Declaration of Helsinki (as revised in 2013), and was approved by the Institutional Ethics Committee of Xinhua Hospital affiliated to Shanghai Jiao Tong University School of Medicine. Individual consent for this retrospective analysis was waived.

Open Access Statement: This is an Open Access article distributed in accordance with the Creative Commons Attribution-NonCommercial-NoDerivs 4.0 International License (CC BY-NC-ND 4.0), which permits the noncommercial replication and distribution of the article with the strict proviso that no changes or edits are made and the original work is properly cited (including links to both the formal publication through the relevant DOI and the license). See: https://creativecommons.org/licenses/by-nc-nd/4.0/.

\section{References}

1. Naidich DP, Bankier AA, MacMahon H, Schaefer-Prokop CM, Pistolesi M, Goo JM, Macchiarini P, Crapo JD, Herold CJ, Austin JH, Travis WD. Recommendations for the management of subsolid pulmonary nodules detected at CT: a statement from the Fleischner Society. Radiology 2013;266:304-17.

2. Lee HY, Choi YL, Lee KS, Han J, Zo JI, Shim YM, Moon JW. Pure ground-glass opacity neoplastic lung nodules: histopathology, imaging, and management. AJR Am J Roentgenol 2014;202:W224-33.

3. Lee SM, Park CM, Goo JM, Lee HJ, Wi JY, Kang CH. Invasive pulmonary adenocarcinomas versus preinvasive lesions appearing as ground-glass nodules: differentiation by using CT features. Radiology 2013;268:265-73.

4. Heidinger BH, Anderson KR, Nemec U, Costa DB, Gangadharan SP, VanderLaan PA, Bankier AA. Lung adenocarcinoma manifesting as pure ground-glass nodules: 
correlating CT size, volume, density, and roundness with histopathologic invasion and size. J Thorac Oncol 2017;12:1288-98.

5. MacMahon H, Naidich DP, Goo JM, Lee KS, Leung ANC, Mayo JR, Mehta AC, Ohno Y, Powell CA, Prokop M, Rubin GD, Schaefer-Prokop CM, Travis WD, Van Schil PE, Bankier AA. Guidelines for management of incidental pulmonary nodules detected on CT images: from the Fleischner Society 2017. Radiology 2017;284:228-43.

6. Bankier AA, MacMahon H, Goo JM, Rubin GD, Schaefer-Prokop CM, Naidich DP. Recommendations for measuring pulmonary nodules at CT: a statement from the Fleischner Society. Radiology 2017;285:584-600.

7. Bai C, Choi CM, Chu CM, Anantham D, Chung-Man Ho J, Khan AZ, Lee JM, Li SY, Saenghirunvattana S, Yim A. Evaluation of pulmonary nodules: clinical practice consensus guidelines for Asia. Chest 2016;150:877-93.

8. Callister ME, Baldwin DR, Akram AR, Barnard S, Cane P, Draffan J, Franks K, Gleeson F, Graham R, Malhotra P, Prokop M, Rodger K, Subesinghe M, Waller D, Woolhouse I; British Thoracic Society Pulmonary Nodule Guideline Development Group; British Thoracic Society Standards of Care Committee. British Thoracic Society guidelines for the investigation and management of pulmonary nodules. Thorax 2015;70:ii1-54.

9. Blasberg JD, Pass HI, Donington JS. Sublobar resection: a movement from the Lung Cancer Study Group. J Thorac Oncol 2010;5:1583-93.

10. Tsutani Y, Miyata Y, Nakayama H, Okumura S, Adachi S, Yoshimura M, Okada M. Appropriate sublobar resection choice for ground glass opacity-dominant clinical stage IA lung adenocarcinoma: wedge resection or segmentectomy. Chest 2014;145:66-71.

11. Dai C, Shen J, Ren Y, Zhong S, Zheng H, He J, Xie D, Fei K, Liang W, Jiang G, Yang P, Petersen RH, Ng CS, Liu CC, Rocco G, Brunelli A, Shen Y, Chen C, He J. Choice of surgical procedure for patients with NSCLC $\leq 1$ $\mathrm{cm}$ or $>1$ to $2 \mathrm{~cm}$ among lobectomy, segmentectomy, and wedge resection: a population-based study. J Clin Oncol 2016;34:3175-82.

12. Ishiguro F, Matsuo K, Fukui T, Mori S, Hatooka S, Mitsudomi T. Effect of selective lymph node dissection based on patterns of lobe-specific lymph node metastases on patient outcome in patients with resectable non-small cell lung cancer: a large-scale retrospective cohort study applying a propensity score. J Thorac Cardiovasc Surg 2010;139:1001-6.
13. Haruki T, Aokage K, Miyoshi T, Hishida T, Ishii G, Yoshida J, Tsuboi M, Nakamura H, Nagai K. Mediastinal nodal involvement in patients with clinical stage I nonsmall-cell lung cancer: possibility of rational lymph node dissection. J Thorac Oncol 2015;10:930-6.

14. Wilshire CL, Louie BE, Manning KA, Horton MP, Castiglioni M, Gorden JA, Aye RW, Farivar AS, Vallières E. Radiologic evaluation of small lepidic adenocarcinomas to guide decision making in surgical resection. Ann Thorac Surg 2015;100:979-88.

15. Travis WD, Brambilla E, Noguchi M, Nicholson AG, Geisinger KR, Yatabe $\mathrm{Y}$, et al. International Association for the Study of Lung Cancer/American Thoracic Society/European Respiratory Society international multidisciplinary classification of lung adenocarcinoma. J Thorac Oncol 2011;6:244-85.

16. Nemec U, Heidinger BH, Anderson KR, Westmore MS, VanderLaan PA, Bankier AA. Software-based risk stratification of pulmonary adenocarcinomas manifesting as pure ground glass nodules on computed tomography. Eur Radiol 2018;28:235-42.

17. Si MJ, Tao XF, Du GY, Cai LL, Han HX, Liang XZ, Zhao JM. Thin-section computed tomography-histopathologic comparisons of pulmonary focal interstitial fibrosis, atypical adenomatous hyperplasia, adenocarcinoma in situ, and minimally invasive adenocarcinoma with pure groundglass opacity. Eur J Radiol 2016;85:1708-15.

18. Zhang BW, Zhang Y, Ye JD, Qiang JW. Use of relative CT values to evaluate the invasiveness of pulmonary subsolid nodules in patients with emphysema. Quant Imaging Med Surg 2021;11:204-14.

19. Li Q, Fan L, Cao ET, Li QC, Gu YF, Liu SY. Quantitative CT analysis of pulmonary pure ground-glass nodule predicts histological invasiveness. Eur J Radiol 2017;89:67-71.

20. Han L, Zhang P, Wang Y, Gao Z, Wang H, Li X, Ye Z. CT quantitative parameters to predict the invasiveness of lung pure ground-glass nodules (pGGNs). Clin Radiol 2018;73:504.e1-504.e7.

21. Fu F, Zhang Y, Wang S, Li Y, Wang Z, Hu H, Chen H. Computed tomography density is not associated with pathological tumor invasion for pure ground-glass nodules. J Thorac Cardiovasc Surg 2020. DOI:10.1016/ j.jtcrs.2020.04.169.

22. Chen PA, Huang EP, Shih LY, Tang EK, Chien CC, Wu MT, Wu FZ. Qualitative CT criterion for subsolid nodule subclassification: improving interobserver agreement and pathologic correlation in the adenocarcinoma spectrum. 
Acad Radiol 2018;25:1439-45.

23. Wu FZ, Chen PA, Wu CC, Kuo PL, Tsao SP, Chien CC, Tang EK, Wu MT. Semiquantative visual assessment of sub-solid pulmonary nodules $\leqq 3 \mathrm{~cm}$ in differentiation of lung adenocarcinoma spectrum. Sci Rep 2017;7:15790.

24. Cai J, Liu H, Yuan H, Wu Y, Xu Q, Lv Y, Li J, Fu J, Ye J. A radiomics study to predict invasive pulmonary adenocarcinoma appearing as pure ground-glass nodules. Clin Radiol 2021;76:143-51.

25. Fan L, Fang $M$, Li Z, Tu W, Wang S, Chen W, Tian J, Dong D, Liu S. Radiomics signature: a biomarker for the preoperative discrimination of lung invasive adenocarcinoma manifesting as a ground-glass nodule. Eur Radiol 2019;29:889-97.

26. Li L, Li H, Shu J, Pan J, Chen X, Ying M, Xu Y, Wang $\mathrm{D}$, Pang P. Influences of scan-position on clinical ultrahigh-resolution CT scanning: a preliminary study. Sci Rep 2019;9:1134.

27. Zhu H, Zhang L, Wang Y, Hamal P, You X, Mao H, Li F, Sun $\mathrm{X}$. Improved image quality and diagnostic potential using ultra-high-resolution computed tomography of the lung with small scan FOV: a prospective study. PloS One 2017;12:e0172688.

28. Kakinuma R, Moriyama N, Muramatsu Y, Gomi S, Suzuki M, Nagasawa H, et al. Ultra-high-resolution computed tomography of the lung: image quality of a prototype scanner. PLoS One 2015;10:e0137165.

29. Zhu Y, Hou D, Lan M, Sun X, Ma X. A comparison of ultra-high-resolution CT target scan versus conventional CT target reconstruction in the evaluation of groundglass-nodule-like lung adenocarcinoma. Quant Imaging Med Surg 2019;9:1087-94.

30. Travis WD, Brambilla E, Nicholson AG, Yatabe Y, Austin JHM, Beasley MB, Chirieac LR, Dacic S, Duhig E, Flieder DB, Geisinger K, Hirsch FR, Ishikawa Y, Kerr KM, Noguchi M, Pelosi G, Powell CA, Tsao MS, Wistuba I; WHO Panel. The 2015 World Health Organization classification of lung tumors: impact of genetic, clinical and radiologic advances since the 2004 classification. J Thorac Oncol 2015;10:1243-60.

31. Kakinuma R, Noguchi M, Ashizawa K, Kuriyama K, Maeshima AM, Koizumi N, Kondo T, Matsuguma H, Nitta N, Ohmatsu H, Okami J, Suehisa H, Yamaji T, Kodama K, Mori K, Yamada K, Matsuno Y, Murayama S, Murata K. Natural history of pulmonary subsolid nodules: a prospective multicenter study. J Thorac Oncol 2016;11:1012-28.

32. Revel MP, Mannes I, Benzakoun J, Guinet C, Léger T,
Grenier P, Lupo A, Fournel L, Chassagnon G, Bommart S. Subsolid lung nodule classification: a CT criterion for improving interobserver agreement. Radiology 2018;286:316-25.

33. Chu ZG, Li WJ, Fu BJ, Lv FJ. CT Characteristics for Predicting Invasiveness in Pulmonary Pure Ground-Glass Nodules. AJR Am J Roentgenol 2020;215:351-8.

34. Bankier AA, Estenne M, Kienzl D, Müller-Mang C, Van Muylem A, Gevenois PA. Gravitational gradients in expiratory computed tomography examinations of patients with small airways disease: effect of body position on extent of air trapping. J Thorac Imaging 2010;25:311-9.

35. McDonough JE, Knudsen L, Wright AC, Elliott W, Ochs M, Hogg JC. Regional differences in alveolar density in the human lung are related to lung height. J Appl Physiol 2015;118:1429-34.

36. Pennati F, Salito C, Baroni G, Woods J, Aliverti A. Comparison between multivolume CT-based surrogates of regional ventilation in healthy subjects. Acad Radiol 2014;21:1268-75.

37. Ferretti GR, Arbib F, Roux JF, Bland V, Lantuejoul S. Effect of Lung Volume and Gravity on the Attenuation and Size of a Pure Ground-Glass Nodule. J Thorac Imaging 2012;27:W15-7.

38. Zhang L, Yankelevitz DF, Carter D, Henschke CI, Yip R, Reeves AP. Internal growth of nonsolid lung nodules: radiologic-pathologic correlation. Radiology 2012;263:279-86.

39. Eguchi T, Kondo R, Kawakami S, Matsushita M, Yoshizawa A, Hara D, Matsuoka S, Takeda T, Miura K, Agatsuma H, Sakaizawa T, Tominaga Y, Saito G, Toishi M, Hamanaka K, Hashizume M, Shiina T, Amano J, Koizumi T, Yoshida K. Computed tomography attenuation predicts the growth of pure ground-glass nodules. Lung Cancer 2014;84:242-7.

40. Tamura M, Shimizu Y, Yamamoto T, Yoshikawa J, Hashizume Y. Predictive value of one-dimensional mean computed tomography value of ground-glass opacity on high-resolution images for the possibility of future change. J Thorac Oncol 2014;9:469-72.

41. Zhao Q, Wang JW, Yang L, Xue LY, Lu WW. CT diagnosis of pleural and stromal invasion in malignant subpleural pure ground-glass nodules: an exploratory study. Eur Radiol 2019;29:279-86.

42. Lee KH, Goo JM, Park SJ, Wi JY, Chung DH, Go H, Park HS, Park CM, Lee SM. Correlation between the size of the solid component on thin-section CT and 
the invasive component on pathology in small lung adenocarcinomas manifesting as ground-glass nodules. J Thorac Oncol 2014;9:74-82.

43. Liu Y, Sun H, Zhou F, Su C, Gao G, Ren S, Zhou C,

Cite this article as: Ren H, Liu F, Xu L, Sun F, Cai J, Yu L, Guan W, Xiao H, Li H, Yu H. Predicting the histological invasiveness of pulmonary adenocarcinoma manifesting as persistent pure ground-glass nodules by ultra-high-resolution CT target scanning in the lateral or oblique body position. Quant Imaging Med Surg 2021;11(9):4042-4055. doi: 10.21037/ qims-20-1378
Zhang Z, Shi J. Imaging features of TSCT predict the classification of pulmonary preinvasive lesion, minimally and invasive adenocarcinoma presented as ground glass nodules. Lung Cancer 2017;108:192-7. 